\title{
Soya isoflavone-enriched cereal bars affect markers of endothelial function in postmenopausal women
}

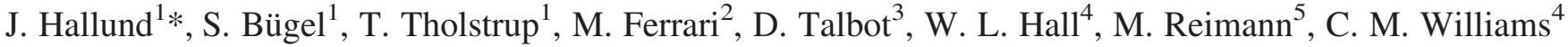 \\ and N. Wiinberg ${ }^{6}$ \\ ${ }^{1}$ Department of Human Nutrition, Centre for Advanced Food Studies, The Royal Veterinary and Agricultural University, \\ Frederiksberg, Denmark \\ ${ }^{2}$ Human Nutrition Unit, National Institute for Research on Food and Nutrition, Rome, Italy \\ ${ }^{3}$ Unilever R\&D, Colworth, Sharnbrook, Bedfordshire, UK \\ ${ }^{4}$ Hugh Sinclair Unit of Human Nutrition, School of Food Biosciences, University of Reading, Reading, UK \\ ${ }^{5}$ Dietary Fibre and the Metabolic Syndrome Group, German Institute of Human Nutrition, Potsdam-Rehbruecke, Nuthetal, \\ Germany \\ ${ }^{6}$ Department of Clinical Physiology and Nuclear Medicine, Frederiksberg University Hospital, Frederiksberg, Denmark
}

(Received 30 August 2005 - Revised 13 December 2005 - Accepted 20 December 2005)

\begin{abstract}
Soya isoflavones are thought to be cardioprotective due to their structural similarity to oestrogen. In order to investigate the effect of soya isoflavones on markers of endothelial function we conducted a randomised, double-blind, placebo-controlled, cross-over study with thirty healthy postmenopausal women. The women consumed cereal bars, with or without soya isoflavones $(50 \mathrm{mg} / \mathrm{d})$, for 8 weeks, separated by an 8 -week washout period. Systemic arterial complince (SAC), isobaric arterial compliance (IAC), flow-mediated endothelium-dependent vasodilation (FMD) and nitroglycerine-mediated endothelium-independent vasodilation (NMD) were measured at the beginning of the study and after each intervention period. Blood pressure (BP) and plasma concentrations of nitrite and nitrate (NOx) and endothelin-1 (ET-1) were measured at the beginning and end of each intervention period. NMD was 13.4 (SEM 2.0) \% at baseline and 15.5 (SEM 1.1) \% after isoflavone treatment compared with 12.4 (SEM 1.0) \% after placebo treatment $(P=0.03$ ). NOx increased from 27.7 (SEM 2.7) to 31.1 (SEM 3.2) $\mu \mathrm{M}$ after isoflavones treatment compared with 25.4 (SEM 1.5) to 20.4 (SEM 1.1) $\mu \mathrm{M}$ after placebo treatment $(P=0.003)$ and a significant increase in the NOx:ET-1 ratio $(P=0.005)$ was observed after the isoflavone treatment compared with placebo. A significant difference in SAC after the isoflavone and placebo treatment was observed $(P=0 \cdot 04)$. No significant difference was found in FMD, IAC, BP and ET-1. In conclusion, 8 weeks' consumption of cereals bars enriched with $50 \mathrm{mg}$ soya isoflavones/d increased plasma NOx concentrations and improved endothelium-independent vasodilation in healthy postmenopausal women.
\end{abstract}

Phyto-oestrogens: Postmenopausal women: Endothelial function: Arterial compliance

Women have an increased risk of CVD after the occurrence of menopause and this has been attributed to the decline in circulating levels of oestrogen (Witteman et al. 1989). The occurrence of menopause is associated with a more atherogenic lipid profile (Jensen et al. 1990) and altered vascular reactivity. Endothelial function, which plays a central role in the development, progression and clinical manifestations of atherosclerosis, has been shown to decline at about the time of menopause (Celermajer et al. 1994) and a similar decline has been reported for the distensibility of the aorta (Waddell et al. 2001).

It has been hypothesised that isoflavones may be cardioprotective due to their structural similarity to $17 \beta$-oestradiol. They have a high binding affinity to the oestrogen receptors, especially oestrogen receptor $\beta$ (Kuiper et al. 1997). The most important dietary sources of isoflavones are genistein and daidzein found in soya ingredients and soya foods, but isoflavones are also found in other plants, such as red clover. The high consumption of soya foods in Asian countries has been suggested to contribute to their lower rates of CVD compared with Western countries. High dietary intake of phyto-oestrogens has been associated with decreased aortic stiffness in postmenopausal women (van der Schouw et al. 2002). A few clinical controlled studies have shown that isoflavone extracts improve systemic arterial compliance (SAC) in healthy postmenopausal women (Nestel et al. 1997, 1999; Teede et al. 2003). The effect on endothelial function has been less consistent. Isolated isoflavones have been

Abbreviations: BP, blood pressure; eNOS, endothelial NO synthase; ET-1, endothelin-1; FMD, flow-mediated endothelium-dependent vasodilation; IAC, isobaric arterial compliance; ISOHEART, isoflavones for reducing risk of CHD among postmenopausal women; NMD, nitroglycerine-mediated endothelium-independent vasodilation; NOx, nitrite and nitrate; SAC, systemic arterial compliance; TMP, transmural pressure.

* Corresponding author: Dr J. Hallund, fax +45 35282483, email jeha@kvl.dk 
shown to improve flow-mediated endothelium-dependent vasodilation (FMD) (Squadrito et al. 2002, 2003) and nitroglycerine-mediated endothelium-independent vasodilation (NMD) (Lissin et al. 2004) of the brachial artery in postmenopausal women. However, a number of studies have shown no effect of isoflavone extract supplementation and have reported FMD to be unaffected (Simons et al. 2000; Teede et al. 2003; Lissin et al. 2004). The mechanisms underlying these potential beneficial effects of isoflavones are not fully understood. It has been suggested that isoflavones may activate the enzyme endothelial NO synthase (eNOS), which synthesises the endothelium-derived relaxing factor $\mathrm{NO}$ from the substrate L-arginine (Liu et al. 2004). Isoflavone supplementation has been shown to increase the sum of serum nitrite and nitrate (NOx) in healthy postmenopausal women (Squadrito et al. 2002, 2003) and to have no effect on NOx (Nikander et al. 2003).

The aim of the present study was to examine whether consumption of isolated soya isoflavones, provided within a food vehicle, would affect endothelial function in healthy postmenopausal women. In the present paper we present vascular endpoint results of endothelial function and arterial compliance. In addition we present results of blood pressure (BP), plasma concentrations of NOx, endothelin-1 (ET-1) and the NOx:ET-1 ratio.

\section{Methods}

\section{Subjects}

Healthy postmenopausal women were recruited from Copenhagen and the surrounding areas through written invitation to individuals found through the central national register and by advertisements in the local media. The women were 57 (SD 5) years and postmenopausal (defined as no menstrual period for $>12$ months and confirmed by follicle-stimulating hormone $(41-124 \mathrm{IU} / \mathrm{l})$ and luteinising hormone $(>14 \mathrm{IU} / \mathrm{l})$ if $<36$ months had elapsed since the last menstrual period). Before the study none of the women had used hormone replacement therapy in the previous 6 months, or fatty acid-, isoflavone-, vitamin- or mineral-containing supplements in the previous 2 months, or antibiotics in the previous 3 months. None of the women had any history of diabetes, inflammatory diseases or CVD, and they did not use antihypertensive, anti-inflammatory or lipid-lowering drugs on a regular basis. Renal and liver functions were normal at entry. All women were non-smokers, had a BP of less than 160/90 mmHg and a BMI between 20 and $32 \mathrm{~kg} /$ $\mathrm{m}^{2}$. A screening blood sample was taken before entry and all women had a total cholesterol $<8 \mathrm{mmol} / 1$, triacylglycerols $1-3 \mathrm{mmol} / \mathrm{l}, \mathrm{HDL}<1.9 \mathrm{mmol} / \mathrm{l}, \mathrm{Hb}>7.4 \mathrm{mmol} / \mathrm{l}$, and fasting glucose $<6.5 \mathrm{mmol} / \mathrm{l}$. A total of thirty women were included in the study. All participating women were stratified according to age, BMI and triacylglycerols before study entry and randomised into two groups. Two women withdrew from the study due to the initiation of hormone replacement therapy and personal reasons. Twenty-eight women completed the study according to the protocol. Ethical approval was obtained from the Local Research Ethical Committee of Copenhagen and Frederiksberg (KF 01-130/02). All women received oral and written information about the study before they gave written informed consent.

\section{Study design}

The present study is part of the 'Isoflavones for reducing risk of coronary heart disease among postmenopausal women' (ISOHEART) study, which is a multi-centre study conducted in Denmark, Great Britain, Germany and Italy. The main purpose of this randomised, double-blind, placebo-controlled, cross-over, dietary intervention study was to examine the effects of consumption of isolated soya isoflavones, provided within a food vehicle, on a range of vascular risk markers associated with CHD. The women consumed two cereal bars daily, with or without soya isoflavones, for 8 weeks, separated by an 8 -week washout. The women were requested to eat two cereal bars per $d$ in addition to their habitual diet during the intervention periods, one with breakfast and one in the late afternoon or evening. The cereal bars were identical in all respects other than enrichment with isoflavones $(50 \mathrm{mg} / \mathrm{d})$. The soya isoflavone extract used was formulated in a $40 \%$ soya isoflavones concentration and had a genistein:daidzein ratio of 2:1. The content of glycosides was $96 \%$, aglycones $2 \%$ and acetylglucosides $2 \%$ (Solgen 40; Solbar Plant Extracts Ltd, Israel). The cereal bars ( $40 \mathrm{~g}$ ) manufactured by a commercial company in the UK (Efamol Ltd, Manchester, UK) were produced in four different flavours (apricot and almond, apple and cardamom, hazelnut, or raspberry). Each cereal bar contained: energy, $652 \mathrm{~kJ}$; protein, $2.6 \mathrm{~g}$; carbohydrate, $17.3 \mathrm{~g}$; fat, $8.5 \mathrm{~g}$; fibre, $1.8 \mathrm{~g}$; Na, $0.012 \mathrm{~g}$. Compliance was validated from serum concentration and urinary excretion of isoflavones.

The women visited the department on four occasions: at the beginning of the study (week 0); after the first intervention period (week 8); at the beginning of the second intervention period after an 8-week washout period (week 16); after the second intervention period (week 24). The women were instructed to consume a standardised low-fat meal providing a maximum of $10 \mathrm{~g}$ fat the evening before each visit. Height was measured at week 0. Weight, BP and $24 \mathrm{~h}$ urinary isoflavone excretion were measured at weeks $0,8,16$ and 24 . During the morning at weeks 0 , 8,16 and 24 after $15 \mathrm{~min}$ of supine rest, $12 \mathrm{~h}$ fasting blood samples were collected for the analysis of NOx and ET-1. SAC, isobaric arterial compliance (IAC), FMD and NMD were measured at weeks 0,8 and 24. Successful measurements of SAC and IAC were only obtained for twenty-five of the volunteers. Vascular measurements of FMD, NMD, SAC and IAC were performed during the afternoon. The time of the measurements was standardised and each woman was measured at the same time during the afternoon of each visit. A standardised breakfast and lunch were provided on the day of the measurements, which together contained a total of $<7 \mathrm{~g}$ fat, $<25 \mathrm{mg}$ vita$\min \mathrm{C}$ and $<2 \mathrm{mg}$ vitamin $\mathrm{E}$. All the women were instructed to finish the meals at least $3 \mathrm{~h}$ before the measurements and to abstain from coffee, caffeine-containing beverages and tea during the day.

A $3 \mathrm{~d}$ food record was performed 1 week before the study and during the last week of each intervention period to estimate habitual dietary intake. Energy and nutrient intake were calculated using the Dankost 2000 dietary assessment software (National Food Agency, Herlev, Denmark). 


\section{Vascular measurements}

Endothelial function was measured by ultrasound using the method described by Corretti et al. (2002). FMD and NMD were calculated as the change in the diameter of the right superficial brachial artery following ischaemia and sublingual nitroglycerine administration respectively, as a percentage of the baseline diameter. The baseline diameter of the right superficial brachial artery was measured at rest and $1 \mathrm{~min}$ after reactive hyperaemia induced by an arterial occlusion cuff around the proximal part of the forearm to a pressure of $>200 \mathrm{mmHg}$ for $4.5 \mathrm{~min}$ causing FMD. After $10 \mathrm{~min}$ the baseline diameter was measured again and compared with arterial response $4 \mathrm{~min}$ after $400 \mu \mathrm{g}$ nitroglycerine spray administrated sublingually as glyceryl trinitrate causing NMD. The diameter was measured by Q LAB Quantification software (Philips, Andover, MA, USA) using stored images of the brachial artery. Each diameter was measured at two cardiac cycles at the end of diastole, defined by the $\mathrm{R}$ wave. Images were obtained by a B-mode scan on a standard Acuson $128 \mathrm{XP} / 10 \mathrm{c}$ system (Acuson, Mountain View, CA, USA) using a $7-10 \mathrm{MHz}$ linear array transducer while an electrocardiogram trace was simultaneously recorded. Images were magnified using the resolution box function. Arterial flow was measured using a Doppler signal at maximum $70^{\circ}$ to the vessel with the range gate at the same size as the baseline diameter of the artery. Flow measurements were performed at rest and during the first $10-15 \mathrm{~s}$ after cuff deflation. All measurements were performed in a supine position after $15 \mathrm{~min}$ rest at room temperature in a calm environment.

IAC was determined by a volume-oscillometric technique (Artcomp ${ }^{\circledR}$; Critikon, Tampa, FL, USA). Compliance was calculated from the oscillation amplitudes and the arterial pressure independently of the actual BP. Simultaneous changes in $\mathrm{BP}$ and oscillations were registered during pulse wave travel at different transmural pressures (TMP) obtained by decremental deflation of the cuff at brachial level. The pressure recordings were similar to measurements of BP obtained by a common oscillometric BP device and the following assessment of volume changes resembled the method in ordinary plethysmography. The oscillations were transformed into changes of volume and the simultaneous recording of BP allows for the calculation of the arterial compliance as a function of the TMP (Mazhbich, 1983). By integrating the arterial compliance in the known TMP range, the volume-pressure relationship for the artery was found. The distensibility was calculated by dividing the arterial compliance by the volume of the artery. The arterial compliance, volume, and distensibility are given at TMP of 0 and $100 \mathrm{mmHg}$. A standard BP cuff appropriate for the size of the extremity was secured around the upper left arm. Three measurements were performed at 1 min intervals and averaged in order to reduce variation. Measurements were performed with the subjects in a supine position after $15 \mathrm{~min}$ rest at room temperature in a calm environment.

SAC was measured as previously described (Faber et al. 2001) and calculated as the stroke volume divided by pulse pressure. Stroke volume and heart rate were measured by impedance cardiography (Cardioscreen ${ }^{\circledR}$; Medis GmbH, Illemenau, Germany). BP and mean arterial pressure were measured simultaneously on the upper arm by an oscillometric sphygmomanometer. Systemic vascular resistance was determined as mean arterial pressure divided by the cardiac output. Stroke volume was calculated using Bernstein's formula (Mehlsen et al. 1991).

\section{Laboratory measurements}

A fasting blood sample was drawn into a $10 \mathrm{ml}$ sodium heparin tube for the NOx analysis and a $10 \mathrm{ml}$ EDTA tube for the ET-1 analysis. Tubes were centrifuged at $1600 \mathrm{~g}$ for $10 \mathrm{~min}$ at $4^{\circ} \mathrm{C}$ and stored at $-80^{\circ} \mathrm{C}$ until further analysis. The concentration of $\mathrm{NOx}$ in serum was measured by NADPH-dependent nitrate reductase assay (Verdon et al. 1995). ET-1 concentrations were determined using the Parameter human endothelin-1 immunoassay kit (R\&D Systems Europe, Abingdon, UK). Urinary and serum genistein, daidzein and equol were analysed using time-resolved (Delfia) fluorescent immunoassays as previously described (de Pascual-Teresa et al. 2005).

\section{Power calculations}

The number of subjects needed was calculated by the method of least standardised difference. Using a power of $90 \%$ with a significance level of $5 \%$, twenty-six volunteers were sufficient to demonstrate a $20 \%$ difference in arterial compliance and endothelial function.

\section{Statistical analysis}

Data describing the characteristics of the volunteers are summarised as means and standard deviations. Data on the outcome of the study are expressed as means and standard errors of the mean. Data were analysed in Statistical Analysis Systems statistical software package version 8.02 (SAS Institute Inc., Cary, NC, USA) using a mixed model analysis of covariance (PROC MIXED) with treatment (isoflavones treatment or placebo) and period (first or second period) as fixed factors. Baseline values of BMI, age and triacylglycerols were included as fixed factors in the final model if the effect was significant (these were used in the stratified randomisation procedure). Subjects were included as a random factor and baseline measurements as a covariate. Further exploratory investigations of equol producers and non-producers as well as continuous equol production were included in the model. For NOx, ET-1, NOx:ET-1 ratio, BMI and BP the baseline value of each intervention period (week 0 and week 8) was used as a covariate. For FMD, NMD, SAC, systemic vascular resistance, IAC at TMP of $0 \mathrm{mmHg}\left(\mathrm{AC}_{0}\right)$, IAC at TMP of $100 \mathrm{mmHg}\left(\mathrm{AC}_{100}\right)$, arterial distensibility of $0 \mathrm{mmHg}\left(\mathrm{D}_{0}\right)$, arterial distensibility of $100 \mathrm{mmHg}\left(\mathrm{D}_{100}\right)$, arterial volume of $0 \mathrm{mmHg}\left(\mathrm{V}_{0}\right)$ and arterial volume of $100 \mathrm{mmHg}\left(\mathrm{V}_{100}\right)$ the baseline value at the beginning of the study (week 0) was used as a covariate. For $\mathrm{AC}_{0}$ and $\mathrm{AC}_{100}$, the data were logarithmic-transformed to obtain normally distributed residuals. Differences in habitual energy and nutrient intake calculated 1 week before the study and during the last week of each intervention period were analysed using ANOVA. 


\section{Results}

The baseline characteristics of the women were as follows: age 57 (SD 5) years; BMI 24.0 (SD 2.6) kg/m²; total cholesterol 5.98 (SD 0.70) mmol/l; LDL-cholesterol 3.91 (SD 0.69) $\mathrm{mmol} / \mathrm{l}$; HDL-cholesterol 1.53 (SD 0.29) mmol/l; triacylglycerols 1.18 (SD 0.47) mmol/l; systolic BP 126 (SD 18) $\mathrm{mmHg}$; diastolic BP 77 (SD 8) $\mathrm{mmHg}$.

Compliance assessed using study diaries showed that $92 \%$ of all cereal bars were consumed. Urinary daidzein and genistein excretion increased from 155 (SEM 85) and 124 (SEM 15) $\mathrm{ng} / \mathrm{ml}$, respectively, at baseline to 2919 (SEM 273) and 2745 (SEM 239) $\mathrm{ng} / \mathrm{ml}$ respectively, at the end of the isoflavone intervention. Urinary daidzein and genistein excretion was 118 (SEM 19) and 160 (SEM 23) ng/ml, respectively, at baseline and 102 (SEM 26) and 138 (SEM 17) ng/ml respectively, at the end of the placebo intervention. In addition, significantly higher serum concentrations of daidzein (35.4 (SEM 3.1 ) and $9.7($ SEM 0.8$) \mathrm{ng} / \mathrm{ml} ; P<0.001)$ and genistein $(68.0$ (SEM 8.5) and $0.5(\operatorname{SEM~0.2)~} \mathrm{ng} / \mathrm{ml} ; P<0.001)$ were found after isoflavone treatment compared with placebo treatment, which demonstrated that compliance was good.

The endothelial-independent vasodilation was significantly higher after isoflavone treatment compared with placebo treatment (Fig. 1). NMD was 13.4 (SEM 2.0) \% at baseline and 15.5 (SEM 1.1) \% after isoflavone treatment compared with 12.4 (SEM 1.0$) \%$ after placebo treatment $(P=0.03)$. No significant difference in endothelium-dependent vasodilation was found between isoflavone and placebo treatment. FMD was 2.2 (SEM 0.8) $\%$ at baseline and 4.3 (SEM 0.9) $\%$ after the isoflavone treatment compared with 3.7 (SEM 0.6) \% after placebo treatment $(P=0 \cdot 54)$. No significant difference was found in the increased flow following cuff occlusion between isoflavone and placebo treatment $(P=0 \cdot 22)$. No difference was observed in the diameter of the brachial artery between the two intervention periods $(P=0.72)$.

A significantly higher NOx concentration $(P=0.003)$ and NOx:ET-1 ratio $(P=0.005)$ were seen after the isoflavone treatment compared with placebo treatment (Table 1). A rise in ET-1 concentration was observed during the placebo

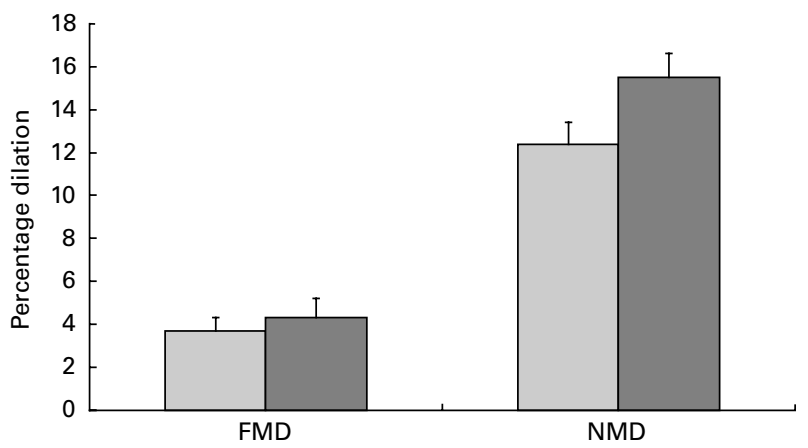

Fig. 1. Flow-mediated endothelium-dependent vasodilation (FMD) and nitroglycerine-mediated endothelium-independent vasodilation (NMD) of the brachial artery after 8 weeks of the isoflavone intervention $(\square)$ and placebo $(\square)$ periods $(n 28)$. Values are means, with their standard errors represented by vertical bars. No significant difference was observed in FMD between isoflavone and placebo treatment $(P=0.54)$. A significant difference was observed in NMD between isoflavone and placebo treatment $(P=0.03)$ (mixed model analysis of covariance). For details of subjects and procedures, see p. 1121. treatment, but no significant difference was observed in ET-1 concentrations between isoflavone and placebo treatment $(P=0 \cdot 28)$.

The SAC, systemic vascular resistance, $\mathrm{AC}_{0}, \mathrm{AC}_{100}, \mathrm{D}_{0}$, $\mathrm{D}_{100}, \mathrm{~V}_{0}$ and $\mathrm{V}_{100}$ after isoflavone and placebo treatment are shown in Table 2. A significant difference in the response to isoflavones and placebo treatment was observed for arterial compliance $(P=0.04)$, which was caused by a higher SAC following the placebo treatment period. No significant differences were observed for any of the other variables.

Volunteers were defined as 'equol producers' if their urinary equol concentration in a $24 \mathrm{~h}$ urine sample during the isoflavone treatment arm was $>936 \mathrm{nmol} / \mathrm{l}$ (Rowland et al. 2000). This approximates to a urinary yield of $>0.45 \mathrm{mg} / \mathrm{d}$. Using these cut-offs, six out of twenty-eight subjects $(21.4 \%)$ were classified as equol producers. Exploratory analysis showed that there were no differences in vascular response to isoflavones and placebo between equol producers and non-equol producers (data not shown). In addition, further exploratory analysis showed that there were no significant interaction between continuous equol production and treatment vascular response to isoflavones and placebo treatment (data not shown).

There were no significant differences between estimated energy, carbohydrate, protein, fibre, fat, saturated fatty acid, MUFA, PUFA and cholesterol intakes during the isoflavone treatment compared with placebo treatment (data not shown). Information on estimated nutrient intakes at baseline and during the isoflavones and placebo period have been published elsewhere in more detail (Hall et al. 2005). In addition, no significant changes in BMI $(P=0.09)$, systolic BP $(P=0.87)$ and diastolic BP $(P=0.71)$ were observed when comparing the two intervention periods. BMI was 24.0 (SD $0.5) \mathrm{kg} / \mathrm{m}^{2}$ at baseline and $24.0(\mathrm{SD} 0.5) \mathrm{kg} / \mathrm{m}^{2}$ at the end of the isoflavone treatment compared with $23.9(\mathrm{SD} 0 \cdot 5) \mathrm{kg} / \mathrm{m}^{2}$ at baseline and $24.1(\mathrm{SD} 0.5) \mathrm{kg} / \mathrm{m}^{2}$ at the end of the placebo treatment. BP was 123 (SD 3)/75 (SD 1) $\mathrm{mmHg}$ at baseline and 121 (SD 3)/75 (SD 1) $\mathrm{mmHg}$ at end of the isoflavone treatment compared with 124 (SD 3)/76 (SD 1) $\mathrm{mmHg}$ at baseline and 121 (SD 3)/75 (SD 1) $\mathrm{mmHg}$ at the end of the placebo treatment.

\section{Discussion}

We have shown that daily consumption of two isoflavoneenriched cereal bars for 8 weeks significantly increased NMD, plasma NOx concentrations and the NOx:ET-1 ratio compared with placebo cereal bars without having an effect on FMD.

Some issues about the methodology used in the study need to be addressed before the results are discussed. To begin with, the number of women included in the present study was calculated using the method of least standardised difference (Senn, 2002) and the study was designed to demonstrate a $20 \%$ difference in FMD using a power of $90 \%$ with a significance level of $5 \%$. Based on the results from the study, this corresponds to an improvement in FMD from 3.7 to $4.4 \%$. It has earlier been suggested that a mean improvement in FMD of at least 2 percentage point would be required to detect a treatment benefit (Sorensen et al. 1995). Post hoc power calculations based on the results from the present study have 
Table 1. Plasma levels of the sum of serum nitrite and nitrate (NOx) and endothelin-1 (ET-1), and NOx:ET-1-ratio at baseline (week 0 ) and week 8 of the intervention and placebo periods $(n$ 28)

(Mean values with their standard errors)

\begin{tabular}{|c|c|c|c|c|c|c|c|c|c|}
\hline \multirow[b]{3}{*}{ Variable } & \multicolumn{4}{|c|}{ Isoflavone intervention } & \multicolumn{4}{|c|}{ Placebo } & \multirow[b]{3}{*}{$P^{*}$} \\
\hline & \multicolumn{2}{|c|}{ Baseline } & \multicolumn{2}{|c|}{ Week 8} & \multicolumn{2}{|c|}{ Baseline } & \multicolumn{2}{|c|}{ Week 8} & \\
\hline & Mean & SEM & Mean & SEM & Mean & SEM & Mean & SEM & \\
\hline $\mathrm{NOx}(\mu \mathrm{m})$ & $27 \cdot 7$ & $2 \cdot 7$ & $31 \cdot 1$ & 3.2 & 25.4 & 1.5 & $20 \cdot 4$ & $1 \cdot 1$ & 0.003 \\
\hline ET-1 (pg/ml) & $1 \cdot 23$ & 0.06 & $1 \cdot 24$ & 0.07 & $1 \cdot 10$ & 0.05 & $1 \cdot 27$ & 0.08 & $0 \cdot 28$ \\
\hline NOx:ET-1 ratio & $25 \cdot 1$ & $3 \cdot 3$ & $31 \cdot 1$ & 5.5 & 24.8 & $1 \cdot 8$ & 18.5 & 1.9 & 0.005 \\
\hline
\end{tabular}

${ }^{*} P$ values are shown for the treatment effect analysed using a mixed model analysis of covariance.

For details of subjects and procedures, see p. 1121.

shown that inclusion of twenty-nine women was sufficient to detect an improvement in FMD of at least 2 percentage point. However, a 2 percentage point improvement in FMD corresponds to an approximately 55 percentage point change in the present study. Moreover, we found no differences in the response to isoflavones and placebo treatment between equol producers and non-producers. It should be noted that the study was not designed to test these differences and that the effect statistical analysis was purely exploratory. However, we have recently published results from the whole ISOHEART study conducted among 117 postmenopausal women, which showed no differences in response to isoflavones between equol producers and non-producers for inflammatory markers (Hall et al. 2005). In addition, results from another recently published study found no significant differences on FMD and BP between equol producers and non-producers after 12 months' intervention with soya protein, even though there was a tendency towards improved FMD and BP among the equol producers (Kreijkamp-Kaspers et al. 2005).

The results presented in the present study support the hypothesis that isoflavones may have an endothelial-independent effect on vascular tissue (Figtree et al. 2000). The present results are similar to the findings published by Lissin et al. (2004), who reported a significant endothelium-independent effect but no endothelium-dependent effect of soya isoflavone extracts. In the present study it was unexpected that the endothelium-independent effect of isoflavones on vascular smooth muscle relaxation was not reflected in a higher FMD. It could be speculated that the discrepancy between the effect on NMD and FMD was due to a changed resting diameter of the brachial artery before and after ischaemia, but no difference was found.

Only a few studies have investigated the effect of isolated soya isoflavones on endothelial function among postmenopausal women and results have been inconsistent. Some studies have reported unaffected FMD after 6 to 8 weeks of isolated red clover isoflavones (Teede et al. 2003) or soya isoflavone supplementation (Simons et al. 2000; Lissin et al. 2004); others have reported improved FMD after 6 and 12 months of pure genistein supplementation (Squadrito et al. 2002, 2003). Isoflavones have been found to improve NMD (Lissin et al. 2004), or to have no effect on NMD (Simons et al. 2000; Squadrito et al. 2002, 2003). Genistein has been shown to cause L-arginine-NO-dependent vasodilation and this effect has been well documented (Walker et al. 2001). However, genistein has also been shown to induce endothelium-independent relaxation that does not involve the endothelium or the eNOS pathway. Figtree et al. (2000) found that

Table 2. Mean values of systemic arterial compliance (SAC), systemic vascular resistance (SVR), isobaric arterial compliance (IAC) and distensibility and volume variables at baseline (week 0 ) and week 8 of the intervention and placebo periods $(n 25)$

(Mean values with their standard errors)

\begin{tabular}{|c|c|c|c|c|c|c|c|}
\hline \multirow[b]{3}{*}{ Variable } & \multirow{2}{*}{\multicolumn{2}{|c|}{ Baseline }} & \multicolumn{4}{|c|}{ Week 8} & \multirow[b]{3}{*}{$P^{\star}$} \\
\hline & & & \multicolumn{2}{|c|}{ Isoflavone intervention } & \multicolumn{2}{|c|}{ Placebo } & \\
\hline & Mean & SEM & Mean & SEM & Mean & SEM & \\
\hline $\mathrm{SAC}(\mathrm{ml} / \mathrm{mmHg})$ & 1.67 & 0.09 & 1.67 & 0.09 & $1 \cdot 76$ & 0.11 & 0.04 \\
\hline $\begin{array}{l}\text { SVR }\left(\text { dynexs } / \mathrm{cm}^{5}\right) \\
\text { IAC }\end{array}$ & 1260 & 75 & 1292 & 65 & 1250 & 64 & 0.06 \\
\hline $\mathrm{AC}_{100}(\mu \mathrm{l} / \mathrm{mmHg}$ per $10 \mathrm{~cm})$ & 1.04 & 0.06 & 1.00 & 0.06 & 0.99 & 0.06 & 0.84 \\
\hline $\mathrm{D}_{0}\left(10^{-3} / \mathrm{mmHg}\right)$ & $27 \cdot 0$ & 0.8 & $27 \cdot 4$ & $1 \cdot 1$ & $28 \cdot 6$ & 1.4 & 0.11 \\
\hline $\mathrm{D}_{100}\left(10^{-3} / \mathrm{mmHg}\right)$ & 0.90 & 0.04 & 0.86 & 0.04 & 0.84 & 0.04 & 0.51 \\
\hline $\operatorname{Vol}_{0}(\mu \mathrm{l} / 10 \mathrm{~cm})$ & 562 & 26 & 602 & 31 & 594 & 27 & 0.98 \\
\hline $\operatorname{Vol}_{100}(\mu \mathrm{l} / 10 \mathrm{~cm})$ & 1147 & 54 & 1183 & 59 & 1181 & 60 & 0.67 \\
\hline
\end{tabular}

$\mathrm{AC}_{0}$, arterial compliance of $0 \mathrm{mmHg} ; \mathrm{AC}_{100}$, arterial compliance of $100 \mathrm{mmHg} ; \mathrm{D}_{0}$, arterial distensibility of $0 \mathrm{mmHg}$; $\mathrm{D}_{100}$, arterial distensibility of $100 \mathrm{mmHg}$; $\mathrm{V}_{0}$, arterial volume of $0 \mathrm{mmHg} ; \mathrm{V}_{100}$, arterial volume of $100 \mathrm{mmHg}$.

${ }^{*} P$ values are shown for the treatment effect analysed using a mixed model analysis of covariance.

For details of subjects and procedures, see p. 1121. 
the relaxing effect of genistein on rabbit coronary arteries was maintained after removal of the endothelium and after inhibition of the eNOS pathway. The suggested mechanism of genistein action is that it appears to function as a $\mathrm{Ca}$ channel blocker in vascular smooth muscle (Figtree et al. 2000; Torregrosa et al. 2003).

The significantly higher NOx concentration and higher NOx:ET-1 ratio after intake of isoflavone-enriched cereal bars are in accordance with changes in plasma concentrations of NOx after genistein supplementation reported by others (Squadrito et al. 2002, 2003), but in contrast to the findings of other studies (Nikander et al. 2003). The present results support the hypothesis that isoflavones affect endothelial function through improved NO availability. It has recently been shown that concentrations of genistein and daidzein, which are close to normal physiological levels, directly induce eNOS activation and NO production in vitro through a mechanism mediated by protein kinase A activation (Liu et al. 2004). Other suggested mechanisms are related to the antioxidant potential of isoflavones, which may prevent superoxidemediated degradation of $\mathrm{NO}$.

$\mathrm{NO}$ is rapidly oxidised into nitrite $\left(\mathrm{NO}^{2-}\right)$ and nitrate $\left(\mathrm{NO}^{3-}\right)$, which are vasodilator inactive at physiological concentrations (Lauer et al. 2001). Due to the rapid conversion and distribution of $\mathrm{NO}$, endogenous $\mathrm{NO}$ production is commonly assessed by measuring the sum of serum nitrite and nitrate (NOx) in plasma. However, plasma NOx concentration may be influenced by NO-independent factors such as dietary intake, and bacterial and enzymic formation (Tannenbaum, 1979). The use of NOx to assess eNOS activity and NO production has been challenged (Lauer et al. 2001). Lauer et al. (2001) showed that plasma levels of nitrite reflect acute changes in eNOS activity, whereas nitrate and NOx did not change during activation of the NO pathway. It is therefore possible that changes in NOx may not necessarily reflect altered eNOS activity and may be caused by reduced NO degradation. However, more recent studies have shown that nitrite may be reduced back into bioactive NO in blood and tissue (Lundberg \& Weitzberg, 2005). This suggests that plasma concentration of NOx may function as a pool for $\mathrm{NO}$, and that the NOx concentration may be important for vascular function and health.

The majority of studies have investigated the short-term effect of isoflavones on endothelial function and the two long-term studies by Squadrito and colleagues are therefore of particular interest (Squadrito et al. 2002, 2003). These studies demonstrated a clear effect of isolated isoflavones on FMD, NOx and ET-1 after 6 and 12 months of supplementation with isolated genistein. However, a recently published study found no improvement in FMD after a 12-month intervention period with soya protein providing $99 \mathrm{mg}$ isoflavones/d (Kreijkamp-Kaspers et al. 2005). Long-term studies are needed in order to fully understand the effect of isoflavones on risk markers associated with CHD. Furthermore, it is likely that different isoflavones may have different effects on vascular markers of endothelial function.

The significant difference in SAC at the end of the isoflavone and placebo treatment period was unexpected and not in accordance with earlier studies (Nestel et al. 1997, 1999; Teede et al. 2003). In addition, there was a tendency towards a lower IAC at TMP of $0 \mathrm{mmHg}$ and a higher systemic vascular resistance after intake of isoflavone-enriched cereal bars compared with intake of control cereal bars. The results of SAC and IAC were obtained from two independent measurements of arterial compliance. Both measurements were observer independent. However, it should be noted that the effect on SAC was caused by a slight, inexplicable increase in SAC after intake of the placebo cereal bars. Earlier studies have reported that red clover isoflavones and soya isoflavones improve SAC (Nestel et al. 1997, 1999; Teede et al. 2003). Teede et al. (2003) showed that the effect on SAC and pulse wave velocity was limited to the group of subjects receiving formononetin, a methylated precursor of daidzein not found in soya. Their results indicate that the source of isoflavones is important. The dose of soya isoflavones used in the present study was lower than in the study by Nestel et al. (1997), who used $80 \mathrm{mg}$ soya isoflavones/d with $35 \mathrm{mg}$ daidzein/d and $45 \mathrm{mg}$ genistein/d. In addition, one-third of the women in the study by Nestel et al. (1997) were perimenopausal and it was among these women that the largest changes in SAC were observed. It is therefore likely that the study population and the different source and doses of isoflavones may explain the difference in SAC between the different studies. The results of the present study do not suggest a beneficial effect of isoflavones on vascular markers of arterial compliance.

In conclusion, we found that 8 weeks' consumption of isoflavone-enriched cereal bars affected vascular reactivity in a group of postmenopausal women. Isoflavones seem to positively affect endothelial function through an endothelium-independent vasodilation of the smooth muscles of the brachial artery as well as through an improved availability of NO. However, a beneficial effect of isoflavones was not observed for endothelium-dependent vasodilation of the brachial artery or arterial compliance. Further studies are needed to improve our understanding of how isoflavones affect the vascular tree.

\section{Acknowledgements}

The present study was carried out with financial support from the Commission of the European Communities, ISOHEART QLK1-2001-00221. It does not necessarily reflect the Commission's views, nor does it in any way anticipate the Commission's future policy in this area.

\section{References}

Celermajer DS, Sorensen KE, Spiegelhalter DJ, Georgakopoulos D, Robinson J \& Deanfield JE (1994) Aging is associated with endothelial dysfunction in healthy men years before the age-related decline in women. J Am Coll Cardiol 24, 471-476.

Corretti et al. (2002).

de Pascual-Teresa S, Hallund J, Talbot D, Schroot J, Williams CM, Bugel S \& Cassidy A (2005) Absorption of isoflavones in humans: effects of food matrix and processing. $J$ Nutr Biochem 17, 257-264.

Faber J, Wiinberg N, Schifter S \& Mehlsen J (2001) Haemodynamic changes following treatment of subclinical and overt hyperthyroidism. Eur J Endocrinol 145, 391-396. 
Figtree GA, Griffiths H, Lu YQ, Webb CM, MacLeod K \& Collins P (2000) Plant-derived estrogens relax coronary arteries in vitro by a calcium antagonistic mechanism. J Am Coll Cardiol 35, $1977-1985$.

Hall WL, Vafeiadou K, Hallund J, et al. (2005) Soy isoflavone enriched foods and inflammatory biomarkers of cardiovascular risk in postmenopausal women: interactions with genotype and equol production. Am J Clin Nutr 82, 1260-1268.

Jensen J, Nilas L \& Christiansen C (1990) Influence of menopause on serum-lipids and lipoproteins. Maturitas 12, 321-331.

Kreijkamp-Kaspers S, Kok L, Bots ML, Grobbee DE, Lampe JW \& van der Schouw YT (2005) Randomized controlled trial of the effects of soy protein containing isoflavones on vascular function in postmenopausal women. Am J Clin Nutr 81, 189-195.

Kuiper GG, Carlsson B, Grandien K, Enmark E, Haggblad J, Nilsson S \& Gustafsson JA (1997) Comparison of the ligand binding specificity and transcript tissue distribution of estrogen receptors alpha and beta. Endocrinology 138, 863-870.

Lauer T, Preik M, Rassaf T, Strauer BE, Deussen A, Feelisch M \& Kelm M (2001) Plasma nitrite rather than nitrate reflects regional endothelial nitric oxide synthase activity but lacks intrinsic vasodilator action. Proc Natl Acad Sci USA 98, 12814-12819.

Lissin LW, Oka R, Lakshmi S \& Cooke JP (2004) Isoflavones improve vascular reactivity in post-menopausal women with hypercholesterolemia. Vasc Med 9, 26-30.

Liu D, Homan LL \& Dillon JS (2004) Genistein acutely stimulates nitric oxide synthesis in vascular endothelial cells by a cyclic adenosine $5^{\prime}$-monophosphate-dependent mechanism. Endocrinology 145, 5532-5539.

Lundberg JO \& Weitzberg E (2005) NO generation from nitrite and its role in vascular control. Arterioscler Thromb Vasc Biol 25, 915-922.

Mazhbich BI (1983) Noninvasive determination of elastic properties and diameter of human limb arteries. Pflugers Arch 396, 254-259.

Mehlsen J, Bonde J, Stadeager C, Rehling M, Tango M \& TrapJensen J (1991) Reliability of impedance cardiography in measuring central haemodynamics. Clin Physiol 11, 579-588.

Nestel PJ, Pomeroy S, Kay S, Komesaroff P, Behrsing J, Cameron JD \& West L (1999) Isoflavones from red clover improve systemic arterial compliance but not plasma lipids in menopausal women. J Clin Endocrinol Metab 84, 895-898.

Nestel PJ, Yamashita T, Sasahara T, Pomeroy S, Dart A, Komesaroff P, Owen A \& Abbey M (1997) Soy isoflavones improve systemic arterial compliance but not plasma lipids in menopausal and perimenopausal women. Arterioscler Thromb Vasc Biol 17, 3392-3398.

Nikander E, Metsa-Heikkila M, Tiitinen A \& Ylikorkala O (2003) Evidence of a lack of effect of a phytoestrogen regimen on the levels of C-reactive protein, E-selectin, and nitrate in postmenopausal women. J Clin Endocrinol Metab 88, 5180-5185.

Rowland IR, Wiseman H, Sanders TA, Adlercreutz H \& Bowey EA (2000) Interindividual variation in metabolism of soy isoflavones and lignans: influence of habitual diet on equol production by the gut microflora. Nutr Cancer 36, 27-32.

Senn S (2002) Cross-over Trials in Clinical Research, 2nd ed. Chichester, UK: John Wiley \& Sons Ltd.

Simons LA, von Konigsmark M, Simons J \& Celermajer DS (2000) Phytoestrogens do not influence lipoprotein levels or endothelial function in healthy postmenopausal women. Am J Cardiol 85, 1297-1301.

Sorensen KE, Celermajer DS, Spiegelhalter DJ, Georgakopoulos D, Robinson J, Thomas O \& Deanfield JE (1995) Non-invasive measurement of human endothelium dependent arterial responses: accuracy and reproducibility. Br Heart J 74, 247-253.

Squadrito F, Altavilla D, Morabito N, et al. (2002) The effect of the phytoestrogen genistein on plasma nitric oxide concentrations, endothelin-1 levels and endothelium dependent vasodilation in postmenopausal women. Atherosclerosis 163, 339-347.

Squadrito F, Altavilla D, Crisafulli A, et al. (2003) Effect of genistein on endothelial function in postmenopausal women: a randomized, double-blind, controlled study. Am J Med 114, 470-476.

Tannenbaum SR (1979) Nitrate and nitrite: origin in humans. Science 205, 1334-1337, 1332.

Teede HJ, McGrath BP, DeSilva L, Cehun M, Fassoulakis A \& Nestel PJ (2003) Isoflavones reduce arterial stiffness: a placebo-controlled study in men and postmenopausal women. Arterioscler Thromb Vasc Biol 23, 1066-1071.

Torregrosa G, Burguete MC, Perez-Asensio FJ, Salom JB, Gil JV \& Alborch E (2003) Pharmacological profile of phytoestrogens in cerebral vessels: in vitro study with rabbit basilar artery. Eur J Pharmacol 482, 227-234.

van der Schouw YT, Pijpe A, Lebrun CE, Bots ML, Peeters PH, van Staveren WA, Lamberts SW \& Grobbee DE (2002) Higher usual dietary intake of phytoestrogens is associated with lower aortic stiffness in postmenopausal women. Arterioscler Thromb Vasc Biol 22, 1316-1322.

Verdon CP, Burton BA \& Prior RL (1995) Sample pretreatment with nitrate reductase and glucose-6-phosphate dehydrogenase quantitatively reduces nitrate while avoiding interference by NADP + when the Griess reaction is used to assay for nitrite. Anal Biochem 224, 502-508.

Waddell TK, Dart AM, Gatzka CD, Cameron JD \& Kingwell BA (2001) Women exhibit a greater age-related increase in proximal aortic stiffness than men. J Hypertens 19, 2205-2212.

Walker HA, Dean TS, Sanders TA, Jackson G, Ritter JM \& Chowienczyk PJ (2001) The phytoestrogen genistein produces acute nitric oxide-dependent dilation of human forearm vasculature with similar potency to 17beta-estradiol. Circulation 103, $258-262$.

Witteman JC, Grobbee DE, Kok FJ, Hofman A \& Valkenburg HA (1989) Increased risk of atherosclerosis in women after the menopause. BMJ 298, 642-644. 